\title{
Cooperative Relaying in Multi-Antenna Fixed Relay Networks
}

\author{
Abdulkareem Adinoyi and Halim Yanikomeroglu
}

\begin{abstract}
Space, cost, and signal processing constraints, among others, often preclude the use of multiple antennas at wireless terminals. This paper investigates distributed decodeand-forward fixed relays (infrastructure-based relaying) which are engaged in cooperation in a two-hop wireless network as a means of removing the burden of multiple antennas on wireless terminals.

In contrast to mobile terminals, the deployment of a small number of antennas on infrastructure-based fixed relays is feasible, thus, the paper examines the impact of multiple antennas on the performance of the distributed cooperative fixed relays. Threshold-based maximal ratio combining (MRC) and thresholdbased selection combining (SC) of these multiple antenna signals are studied and analyzed. It is found that the end-to-end (E2E) error performance of a network which has few relays with many antennas is not significantly worse than that which has many relays each with a fewer antennas. Obviously, the former network has a tremendous deployment cost advantage over the latter. It is also observed that the E2E error performance of a network in which the multiple antennas at relays are configured in SC fashion is not significantly worse than that in which MRC is used. For implementation, SC presents a significantly lower complexity and cost than a full-blown MRC. The analysis in this paper uses the versatile Nakagami fading channels in contrast to the Rayleigh model used in most previous works.
\end{abstract}

Index Terms-Cooperative diversity, fading channels, fixed wireless relays, multiple antennas, selection combining, threshold maximal ratio combining.

\section{INTRODUCTION}

$\mathbf{T}$ HE future wireless systems are envisaged to offer ubiquitous high data-rate coverage in large areas. To meet such ambitious demands, fundamental changes in system design and deployment as well as incorporation of advanced signal processing techniques are required to enable novel and effective ways of collection, distribution and utilization of wireless terminals' signals [1], [2].

Multi-antenna techniques are well studied; a number of promises in these schemes are documented in [3]-[5]. Their application to wireless systems often encounters numerous implementation problems. For example, an element spacing of

Manuscript received April 8, 2005; revised December 23, 2005, April 21, 2006, and June 30, 2006; accepted August 8, 2006. The associate editor coordinating the review of this paper and approving it for publication was T. Duman. This work was supported in part by the Natural Sciences \& Engineering Research Council of Canada (NSERC) under participation in project WINNER (Wireless World Initiative New Radio) - www.ist-winner.org. This paper was presented in part at the IEEE Wireless Communications and Networking Conference (WCNC), Las Vegas, Nevada, USA, April 3 - 6, 2006.

The authors are with the Broadband Communications and Wireless Systems (BCWS) Centre, Dept. of Systems and Computer Engineering, Carleton University, 1125 Colonel By Drive, Ottawa, Ontario, K1S 5B6 Canada, (email: \{adinoyi, halim\}@sce.carleton.ca).

Digital Object Identifier 10.1109/TWC.2007.05227. half the carrier wavelength is required to ensure uncorrelated signals. The future wireless terminals are expected to be small and light. The small size feature limits the spatial separation needed by multiple antenna systems for their optimal performance. The lightweight feature limits the power capability and signal processing that the terminals can support. Hence, the deployment of a large number of antennas on wireless terminals is not feasible, at least in some networks. In addition, efficient power utilization continue to be of great priority in wireless terminals. Therefore, novel techniques for exploiting network resources through the cooperation of nodes, known as cooperative diversity or antenna sharing, are being considered. The interest in this area is steadily growing, thus spawning a surge of publications on relaying and cooperative relaying networks in [1], [2], [6]-[15].

The idea behind cooperative communication dates back to the classical and motivating work of Meulen [16] and Cover and El-Gamal [17] on relay channels. Their theoretical exposition assumes full-duplex, and therefore expensive relays. However, upon relaxing this condition, their work provides the important basis for the cooperative schemes and protocols for inexpensive wireless relay networks that have gained prominence recently [1], [2]. In [1], Laneman and Wornell discuss a cooperative protocol for combating multipath fading. This protocol exploits the spatial diversity available among a collection of distributed terminals that assist one another. The work in [2] presents a similar treatment referred to as user cooperation diversity. It also highlights a practical implementation of the cooperation scheme in the framework of CDMA systems.

In the IEEE 802 wireless world framework, a number of working groups are focusing on developing mesh-enabled standards such as IEEE 802.11s - WLAN (Wireless Local Area Network), IEEE 802.15.5 - WPAN (Wireless Personal Area Network), Mesh Networking, IEEE 802.16j - WMAN (Wireless Metropolitan Area Network), and IEEE 802.20 MBWA (Mobile Broadband Wireless Access). In addition to these on-going standardization efforts, various proprietary mesh/relay network solutions in the unlicensed bands are also being developed by the industrial players. The emergence of the relay-enabled standards in the IEEE 802 family is likely to result in much higher interest and activity for relay-based communications. Already, the WINNER project is developing a relay-enabled deployment concept for the next generation broadband mobile radio access. Their relay deployment strategy is expected to integrate wide area and short range scenarios closing the gap between WLAN-type and cellular systems [18]. It is important to note that the 


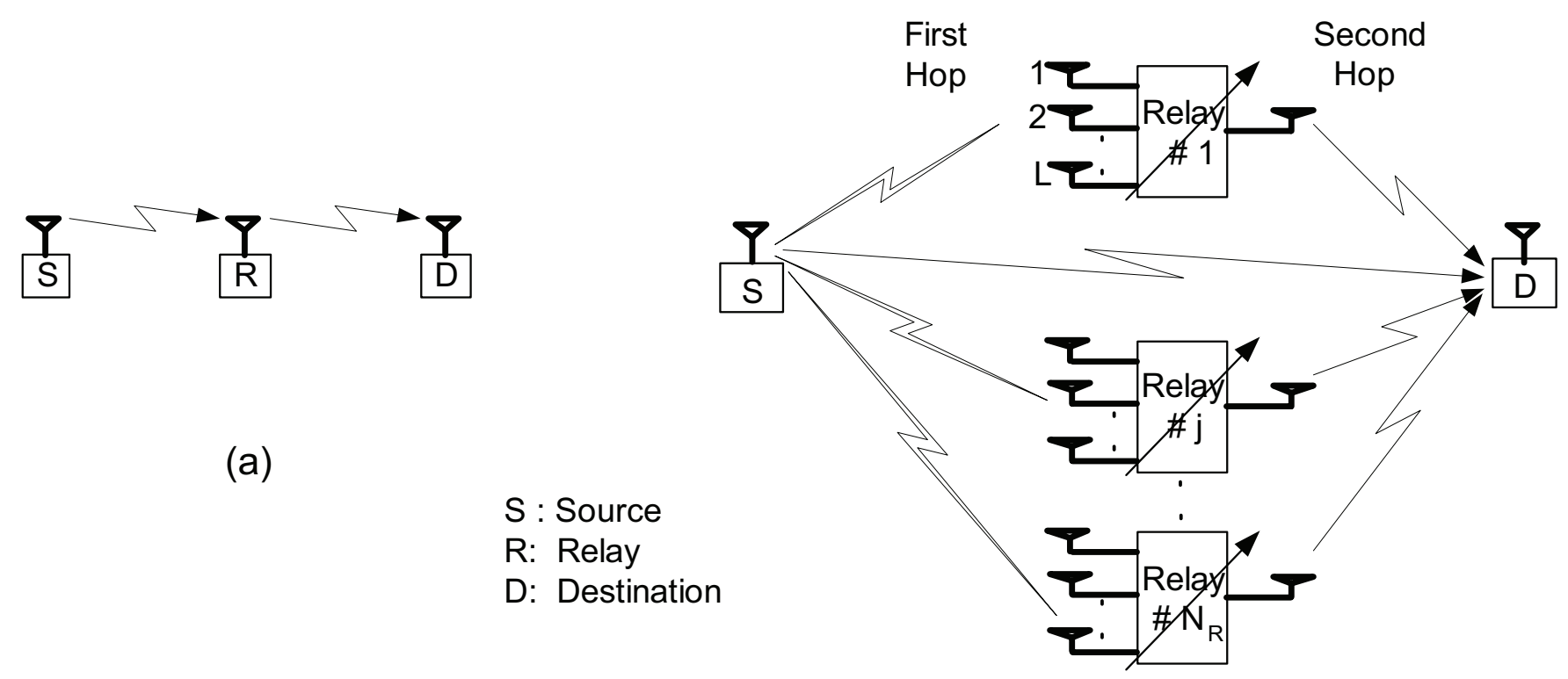

(b)

Fig. 1. Relay networks (a) DF fixed protocol (conventional relaying), (b) TDF protocol with multi-antenna cooperative relays.

WINNER project focuses mainly on infrastructure-based relay deployments.

This paper presents how infrastructure-based fixed relays with/without multiple antennas can be used for providing diversity gains for a wireless terminal which, otherwise, has limitations in the number of antennas that it can bear. Single antenna relaying is a special case of this set-up. Thresholdbased maximal ratio combining (MRC) and threshold-based selection combining (SC) of these multiple antenna signals at the relays are examined and analyzed. Threshold decoding is employed as an additional measure towards combating error propagation. Finally, the paper uses the versatile Nakagami channel model in the analyses in contrast to the Rayleigh fading model which is commonly analyzed. By adopting the Nakagami model the analyses is thus applicable to relaying under a wide variety of channel scenarios, with the Rayleigh channel model serving as a special case. A multi-antenna relay scheme is discussed in [9] but with a number of differences between the present work. Firstly, the relays in [9] do not cooperate but the antennas at each relay do the cooperation. Secondly, the network capacity has been used as their performance criterion.

The following main results are derived. The cooperative diversity strategy employed as an add-on to infrastructurebased relays (which might be deployed primarily for high data rate coverage extension) can provide a small wireless terminals with the advantages of spatial diversity without the need for the physical antennas at these wireless terminals. For a given performance requirement, the multiple antennas at relays can tremendously reduce the number of relays required in a network area, thereby, reducing system deployment cost. Threshold SC at the relays represents an excellent compromise between performance and complexity. It is found that a minimum of two antennas are required at the relay to yield an E2E diversity order equal to the number of relays plus one. Furthermore, it is observed that with a large number of antennas at the relays, threshold decoding is not necessary. However, for a small number of antennas, the choice of threshold becomes crucial.

In the next section the system model, the relaying protocol and the channel model are presented. Section III motivates possible modifications at the relay level that could address the bandwidth inefficiency of repetition-based protocol for multirelay schemes. Following the E2E error performance formulation, some numerical results on decode-and-forward probability of relays are discussed in Section IV. The error probability calculations at the relays are presented in Section V. This is followed by numerical examples and discussions on E2E system performance in Section VI. Finally, conclusions are drawn in Section VII.

\section{The System of Multi-Antenna Multiple Relays}

The conventional fixed protocol decode-and-forward (DF) relay network is shown in Fig. 1(a) where the destination relies only on the signal from the relay. The two-hop system architecture consists of $N_{R}$ threshold decode-and-forward (TDF)-based fixed relays each carrying $L$ diversity antennas (Fig. 1(b)). The scenario referred to as symmetric network, as opposed to the asymmetric one, is considered. The symmetric networks assume that all links (source-relay, relay-destination, and source-destination) experience independent but statistically identical channels with the same mean pathloss.

The employed protocol operates as follows. In the first time slot, the source broadcasts a signal that is received by both destination and relays. The destination stores this signal for future processing. The received $L$ signals at each relay are processed using either SC or MRC diversity technique, depending on the processing complexity that the relays possess. Whether SC or MRC is used, the relay checks the SNR of the received signal against a preset threshold. The relay decodes and forwards only when this SNR is greater than this threshold. 
In the second time slot, the relay either does or does not forward a new (regenerated) signal to destination. If at least one relay forwards, the destination MRC combines the delayed buffered signal received in the first time slot with the new versions from the relays. It is assumed that only one antenna is utilized at each relay for forwarding. The SC detection-based relay requires one receiver chain in contrast to MRC detectionbased relay which requires a separate receiver chain for each antenna [19, pp. 262]. A single transmitting antenna is adopted to keep the cost comparable to a conventional single antenna relay network where one receiver chain is required. Using one transmit antenna provides a basis for fair comparison between a single antenna relaying which uses one transmit antenna. The relays, however, could employ the multiple antennas in an "intelligent" transmit beamforming. Should this be the case, the system performance could be improved which will further increase the attractiveness of multi-antenna relays.

\section{SySTEM COMPLEXITY AND BANDWIDTH PRESERVATION}

A multi-relay scheme employing repetition-based protocol could require enormous bandwidth since each relay requires its own sub-channel in the form of orthogonal time slots for time division multiple access (TDMA) or different frequency slots for frequency division multiple access (FDMA) applications. For a large number of relays, the bandwidth efficiency of such schemes might be so low to the extent that the bandwidth penalty could outweigh the advantages of the multi-relay scheme. To avoid such an inefficient use of the bandwidth, possible modifications on the conventional relays are necessary.

The multi-relay system could use simulcast techniques, where in the second hop the relay set (those with SNR greater than the threshold) simultaneously transmits their signals after being appropriately processed. For instance, the relay could use a finite impulse response (FIR) to filter the input symbols prior to performing a linear-type digital modulation scheme such as MPSK, MQAM, and others. The impulse responses of these relay-embedded filters are different from one relay station to another. However, they are designed to meet the necessary conditions to exploit the maximum diversity gain of MRC. In this case a form of orthogonality is achieved, in terms of filter responses, not in terms of time or frequency slots which are bandwidth-intensive. This transmit modulation diversity strategy is shown to be a fraction of $1 \mathrm{~dB}$ inferior to the classical MRC receive diversity [21]. More details on the filter design can be found in [21], [22].

Another possible strategy is the coherent cooperative transmissions from multiple adjacent nodes proposed in [23], where prior to transmission of the signals, these multiple nodes adjust their transmission characteristics, for example, phases and symbol timings, so that their signals add up coherently at the destination. In fading channels, however, this adjustment is based on the a priori knowledge of the forward channel which can be obtained through feedback from the BS in TDD-based systems.

The modifications (complexities) discussed above may be considered in the deployment of the multi-antenna multi-relay schemes in order to avoid bandwidth expansion involved in the repetition or round-robin transmission protocols. The amount of complexity of relays would depend on how much bandwidth the system designer is prepared to exchange for lower system complexity.

\section{Formulation of System End-TO-End ERror PERFORMANCE}

We define a general link in the network as a node $i$ communicating with another node $j$. A node could correspond to the source, a relay or the destination. The received signal at node $j$ transmitted from node $i$ can then be written as $r_{i j}=r_{i \rightarrow j}=h_{i j} x_{i}+n_{j}$, where $x_{i}$ is the signal emanating from node $i, h_{i j}$ is the channel gain between node $i$ and node $j$ and $n_{j}$ is the additive white Gaussian noise (AWGN). For a relay in a multi-antenna relay network, the input-output relation of the first hop, i.e., source (node $\mathrm{S}$ ) to relays, can be expressed as

$$
\mathbf{r}_{j}=\mathbf{h}_{S j} x_{S}+\mathbf{n}_{j}, \quad j=1,2, \cdots, N_{R},
$$

where $N_{R}$ is the total number of relays, $\mathbf{r}_{j}$ is the $L \times 1$ received vector at the $j$ th relay, $\mathbf{h}_{S j}=\left[h_{S j}^{(1)}, \cdots, h_{S j}^{(L)}\right]^{T}$ is the random channel vector with independent components which are also independent of the components in the $L \times 1$ AWGN noise vector $\mathbf{n}_{j}$. For a coherent detection scheme, perfect recovery of phase and carrier is possible, therefore, each entry $h_{i j}^{(l)}$ represents the magnitude of the fade sample which is assumed follows the Nakagami distribution. It is also assumed that the channel state information for the relay-destination (RD) links is available to the destination whereas, those of the source-relay ( $\mathrm{S}-\mathrm{R}$ ) links are known to the respective relays. The destination does not require any knowledge of the S-R channels.

In the second hop (time-slot), a relay $j$ forwards the preprocessed signal $\left(\hat{x}_{j}\right)$ to the destination (node $\mathrm{D}$ ) provided that the received SNR is greater than a threshold $\gamma_{t h}$. This signal is received at the destination as

$$
r_{j D}=h_{j D} \hat{x}_{j}+w_{j}, \quad j=1, \cdots, N_{R},
$$

where $h_{j D}$ is the fade sample of the link between relay $j$ and destination, and $w_{j}$ is the receiver noise. The model (2) assumes that the signals received at the destination satisfy the orthogonality condition that is the relay signals do not constitute interference. We have assumed the model in (2) in exposing the diversity benefit of the cooperative networks thereby establishing a lower bound on the performance of the system modifications motivated in Section III.

We complete the discussion of this section with the channel model. The analysis assumes that the fading sample has the Nakagami- $m$ distribution, a versatile statistical model that can model a wide range of wireless environments [20]. For instance, the Ricean model which represents the line-of-sight (LOS) communication is captured through the Ricean $K$ parameter to Nakagami- $m$ parameter transformation, $K=$ $\frac{\sqrt{m^{2}-m}}{m-\sqrt{m^{2}-m}}$; moreover, $m=1$ gives the Rayleigh fading model. The SNR per symbol $\gamma_{l}$ at a relay antenna $l$ follows a 
gamma distribution described by ${ }^{1}$

$$
p(\gamma, m)=\frac{m^{m} \gamma^{m-1}}{\bar{\gamma}^{m} \Gamma[m]} \exp \left(-\frac{m \gamma}{\bar{\gamma}}\right), \gamma \geq 0, m \geq 1 / 2
$$

where $\gamma=h_{i j}^{(l) 2} E_{s} / N_{0}$ and $\bar{\gamma}=E\left[h_{i j}^{(l) 2} E_{s} / N_{0}\right]=\Omega E_{s} / N_{0}$ is the average SNR per symbol, $E_{s}$ is the energy per symbol and $\Omega=E\left[h_{i j}^{(l) 2}\right]$. The AWGN is characterized by one-sided power spectral density $N_{0}(\mathrm{~W} / \mathrm{Hz})$ and $\Gamma[\cdot]$ is the gamma function.

\section{A. Error Rate Analysis}

The overall end-to-end (E2E) error rate $P_{e, E 2 E}^{(T D F)}$ of multiantenna multi-relay and protocol can be approximated, using an approach similar to that presented in [24] as

$$
\begin{aligned}
P_{e, E 2 E}^{(T D F)} & =P_{d e c}^{\prime} P_{e, r} P_{e, p}+P_{d e c}^{\prime}\left(1-P_{e, r}\right) P_{e, c o o p} \\
& +\left(1-P_{d e c}^{\prime}\right) P_{e, d i r}
\end{aligned}
$$

The first and second terms represent the component due to cooperation while the third demonstrates the case when cooperation fails. The first term is needed to account for possible error propagation. $P_{e, r}$ is the error probability at the relay given that the received SNR is greater than the threshold, $P_{e, \text { coop }}$ is the destination error rate when cooperation is in effect (i.e., more than one relay paths are combined at destination). $P_{d e c}^{\prime}$ is the probability that at least one relay performs decoding and forwarding, ${ }^{2} P_{e, d i r}$ is the destination probability of error when no relay forwards. The impact of the number of relays have been captured by averaging over the possible cooperative scenarios $P_{e, c o o p}$ and $P_{d e c}^{\prime}$, and therefore, the error probability due to error propagation $P_{e, p}$ can be bounded with the worst scenario, $P_{e, p} \leq 1 / 2$ [24].

The expression in (4) is an approximation for the following reasons: The cumulative impact of the error propagation by the relays (although small in the multi-antenna scheme) is only represented with a bound. The probability of having diversity combining at the destination is modeled with the assumption that at least one relay forwards. To get the exact value we need to consider all possible combinations of forwarding relays, which will only bring computational complexity with no significant impact on the results. This is deduced from the fact that E2E system simulation results match closely with those obtained using this formulation. $P_{d e c}^{\prime}$ is obtained as follows.

Let $\quad P($ relay $r$ does not forward $) \quad=\quad 1$, $P($ relay $r$ does forward $)=1-P_{D F P, r}$. Then, $P_{d e c}^{\prime}$ can be expressed as

$$
\begin{aligned}
P_{d e c}^{\prime} & =1-\prod_{r=1}^{N_{R}}\left(1-P_{D F P, r}\right) \\
& =\sum_{r=1}^{N_{R}}\left(\begin{array}{c}
N_{R} \\
r
\end{array}\right)(-1)^{r+1}\left(P_{D F P}\right)^{r},
\end{aligned}
$$

where $P_{D F P, r}=P_{D F P}$, for all $r$ and $P_{D F P}$ is the decodeand-forward probability (DFP).

\footnotetext{
${ }^{1}$ The $l$ is dropped from the PDF since the SNR at antennas have identical distributions.

${ }^{2}$ This implies there is diversity combining at destination.
}

In symmetric network scenarios assuming that all the relays have the same error performance, then $P_{e, \text { coop }}$ can be evaluated for equal-amplitude modulation as follows: First, it is shown below that the probability of error for a $T$ branch MRC-receiver in Nakagami- $m$ channel is given as $\frac{h \Gamma[T m+1 / 2]}{\sqrt{\pi} \Gamma[T m]} B_{\mu}[T m, 1 / 2]$. The probability that $i$ relays forward which gives rise to $(i+1)$ diversity branches at the destination is given as ${ }^{N_{R}} C_{i}\left(1-P_{D F P}\right)^{N_{R}-i} P_{D F P}^{i}$ where ${ }^{N_{R}} C_{i}=\frac{N_{R} !}{i !\left(N_{R}-i\right) !}$. Therefore, $P_{e, \text { coop }}$ can be expressed by weighted average over the possible cooperation scenarios as

$$
\begin{aligned}
P_{e, \text { coop }} & =\frac{h}{\sqrt{\pi}} \sum_{i=1}^{N_{R}}{ }^{N_{R}} C_{i}\left(1-P_{D F P}\right)^{N_{R}-i} P_{D F P}^{i} \\
& \times \frac{\Gamma[(i+1) m+1 / 2]}{\Gamma[(i+1) m]} B_{\mu}[(i+1) m, 1 / 2]
\end{aligned}
$$

where $B_{\mu}[\cdot, \cdot]$ and $\Gamma[\cdot]$ are the incomplete beta and gamma functions [29], respectively. The parameter $\lambda=g \sin ^{2}(\pi / M)$, $M$ is the modulation constellation size. The parameters $g$ and $h$ are defined according to the modulation scheme and the nature of signal detection. Finally, $\mu=m /(m+\lambda \bar{\gamma})$.

By examining (4) and (6) it can be shown that how well the relay performs has a significant impact on the cooperation benefit. For instance, if $P_{e, r}$ is low and $P_{d e c}^{\prime}$ is high, one obtains the most desirable benefit from the cooperation:

$$
P_{e, E 2 E}^{(T D F)} \approx P_{e, \text { coop }}
$$

The result given in (7) provides considerable information. First, relays always have good and reliable signal to transmit. Then, combining these signals at the destination provides the full diversity benefit. This implies that a diversity order equal to $N_{R}+1$ can be achieved for terminals with one antenna. Furthermore, since the relay perfectly decodes the source information, the source appears (to the destination) as if it were at the position of the relay, hence, path-loss saving advantage can be exploited. This is visible only in the asymmetric channel scenario that incorporates the distancedependent received power variations.

\section{B. Decode-and-Forward Probability Calculation}

The aim in threshold decoding is to ensure that signal forwarded by the relay is reliable since the number of times, reliably detected signal is relayed to the destination has an impact on the cooperation benefit. Therefore, the decodeand-forward probability of relays is an important system performance criterion. Let us now examine the DFP of the multi-antenna relay with threshold DF strategy. We begin with SC where the relay first selects the branch with the largest instantaneous SNR, i.e., $\gamma=\max \left[\gamma_{1}, \cdots, \gamma_{L}\right]$ and then compares it with the set decoding threshold $\gamma_{t h}$. The joint probability density function (PDF) of selecting $n$ largest from $L$ independently and identically distributed random variables is given in a general form in [25] as $p_{\gamma_{1}, \cdots, \gamma_{n}}\left(\gamma_{1}, \cdots, \gamma_{n}\right)=$ $n ! C_{n}^{L}[F(\gamma)]^{L-n} \prod_{l=1}^{n} p\left(\gamma_{l}\right)$, where $F(\gamma)=\int_{0}^{\gamma} p(\xi) d \xi$ is the cumulative distribution function (CDF). Therefore, for the SC-based relaying protocol and the underlying Nakagami$m$ distribution, the required $\mathrm{PDF}$ reduces to $p(\gamma, m)=$ 
$L[F(\gamma, m)]^{L-1} p_{\gamma}(\gamma, m)$ as

$$
\begin{aligned}
p(\gamma, m) & =\frac{L m^{m} \gamma^{m-1} \exp \left(-\frac{m \gamma}{\bar{\gamma}}\right)}{\bar{\gamma}^{m}(\Gamma[m])^{L}} \\
& \times\left(\sum_{k=0}^{\infty} a_{k}\left(\frac{\gamma m}{\bar{\gamma}}\right)^{k+m}\right)^{L-1},
\end{aligned}
$$

where $a_{k}=\frac{(-1)^{k}}{k !(m+k)}$.

We employ the functional series representation to rewrite the series in (8) with the help of $[29$, pp. 19, (0.314)] as

$$
\begin{gathered}
\left(\sum_{k=0}^{\infty} a_{k}\left(\frac{\gamma m}{\bar{\gamma}}\right)^{k+m}\right)^{L-1}= \\
\left(\frac{\gamma m}{\bar{\gamma}}\right)^{m(L-1)} \sum_{p=0}^{\infty} c_{p}\left(\frac{\gamma m}{\bar{\gamma}}\right)^{p},
\end{gathered}
$$

leading to the following PDF

$$
p(\gamma, m)=\frac{L e^{-\frac{m \gamma}{\gamma}}}{(\Gamma[m])^{L} \gamma} \sum_{p=0}^{\infty} c_{p}\left(\frac{m \gamma}{\bar{\gamma}}\right)^{p+m L},
$$

where $c_{0}=m^{1-L}, c_{p}=\frac{m}{p} \sum_{k=1}^{p}(k L-p) a_{k} c_{p-k}, \bar{\gamma}=$ $\Omega_{s r} E_{s} / N_{0}$ and $\Omega_{s r}$ is the average power of source-relay channel.

The derivation of DFP of the selection-based DF relay $P_{D F P, S C}$ can be accomplished by using [29, pp. 356 , (3.351.2)] as

$$
\begin{aligned}
P_{D F P, S C}\left(\gamma_{t h}, m\right) & =\int_{\gamma_{t h}}^{\infty} p_{\gamma}(\xi, m) d \xi \\
& =\frac{L \exp \left(-\frac{m \gamma_{t h}}{\bar{\gamma}}\right)}{(\Gamma[m])^{L}} \sum_{p=0}^{\infty} \sum_{k=0}^{p+m L-1} c_{p} \\
& \times \frac{(p+m L-1) !}{k !}\left(\frac{m \gamma_{t h}}{\bar{\gamma}}\right)^{k} .
\end{aligned}
$$

The infinite summation in the PDF in (8) can be eliminated if integer values of $m$ are strictly considered. Hence, further simplification can be performed leading to an alternative PDF [26]:

$$
\begin{aligned}
p(\gamma, m) & =\frac{L}{(m-1) !} \sum_{l=0}^{L-1}(-1)^{l}\left(\begin{array}{c}
L-1 \\
l
\end{array}\right) \sum_{k=0}^{l(m-1)} b_{k}^{l} \\
& \times\left(\frac{m}{\bar{\gamma}}\right)^{m+k} \gamma^{m+k-1} \exp \left(-(l+1) \frac{m \gamma}{\bar{\gamma}}\right)
\end{aligned}
$$

where $b_{0}^{l}=1, b_{1}^{l}=l, b_{l(m-1)}^{l}=\frac{1}{((m-1) !)^{l}}$, and $b_{k}^{l}=$ $\frac{1}{k} \sum_{j=1}^{\min }[k, m-1] \frac{j(l+1)-k}{j !} b_{k-j}^{l}$ are recursively computed with $k=2,3, \cdots, l(m-1)-1$. With this PDF, a more convenient DFP expression for the selection-based DF relay can be obtained as

$$
\begin{aligned}
P_{D F P, S C}\left(\gamma_{t h}, m\right) & =\int_{\gamma_{t h}}^{\infty} p_{\gamma}(\xi, m) d \xi \\
& =\frac{L}{(m-1) !} \sum_{l=0}^{L-1}(-1)^{l}\left(\begin{array}{c}
L-1 \\
l
\end{array}\right) \\
& \times \sum_{k=0}^{l(m-1)} b_{k}^{l} \exp \left(-\frac{\gamma_{t h}(l+1) m}{\bar{\gamma}}\right) \\
& \times \sum_{t=0}^{m+k-1} \frac{(m+k-1) !}{t !} \frac{\left(m \gamma_{t h} / \bar{\gamma}\right)^{t}}{(l+1)^{m+k-t}}
\end{aligned}
$$

The DFP for the MRC reception at the relay can similarly be derived. In this case, assuming that the branch fading amplitudes are statistically independent and distributed with the Nakagami distribution, then $\gamma$ also follows the Nakagami distribution with a parameter $L m$ [27]. Thus, the following PDF is obtained

$$
p(\gamma, m)=\left(\frac{m}{\bar{\gamma}}\right)^{L m} \frac{\gamma^{L m-1}}{\Gamma[L m]} \exp \left(-\frac{m \gamma}{\bar{\gamma}}\right) .
$$

Consequently, the DFP of MRC-based relay detection can be obtained as

$$
\begin{aligned}
P_{D F P, M R C}\left(\gamma_{t h}, m\right) & =\frac{\exp \left(-\frac{m \gamma_{t h}}{\bar{\gamma}}\right)}{\Gamma[L m]} \\
& \times \sum_{k=0}^{L m-1} \frac{(L m-1) !}{k !}\left(\frac{m \gamma_{t h}}{\bar{\gamma}}\right)^{k} .
\end{aligned}
$$

Fig. 2 shows the improvement in DFP of a relay that is equipped with two antennas $(L=2)$ and SC or MRC detection is utilized. The figure gives curves for different Nakagami parameter $m=1,2,4$, and 6 . The single antenna case is also shown for comparison purposes. Thus, a significant increase in the number of times the relay decodes and forwards is observed for the dual-antenna. This implies that there is an increase in the number of times the destination relies on diversity combining using signals received via the relays. This, however, has to be complemented by the improved error performance at the relays as shown below. These relay performance indicators (relay error rate and probability of decode and forward) are shown to characterize the system E2E performance.

\section{Relay Error Performance Analysis}

In threshold decoding, error performance at the relay implies conditional error. That is, the error performance (residual) given that receiver decodes only when the received SNR is greater than the set threshold. In a clear abuse of notation, to be compact and simple, this conditional dependence will be dropped but should be understood in this context.

We proceed with the derivation of the probability of error at a relay when a threshold is imposed. For a given received SNR $\gamma$, the probability of symbol error for equal amplitude modulation (MPSK) can be expressed as

$$
P_{\mathrm{mpsk}}(e \mid \gamma) \approx h \operatorname{erfc}(\sqrt{\gamma \lambda}),
$$




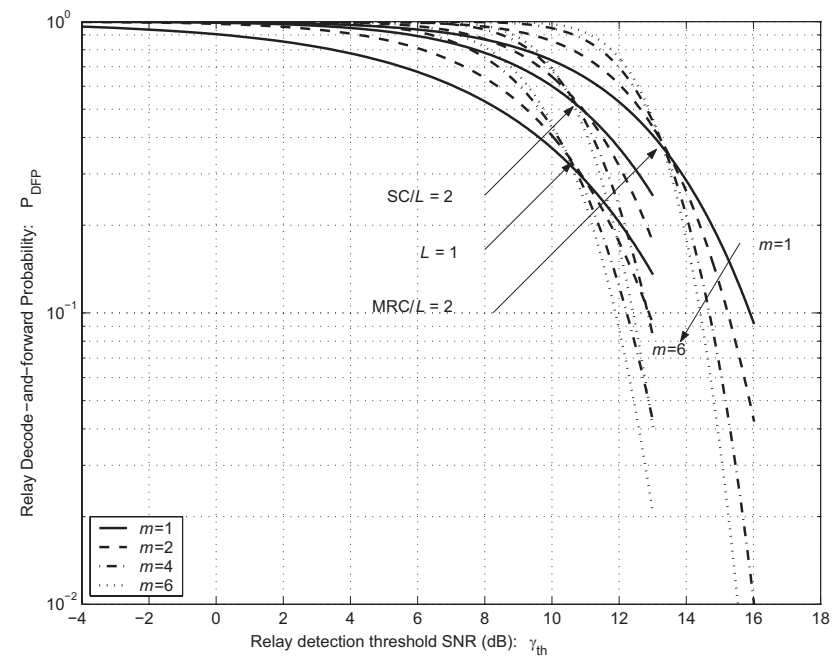

Fig. 2. Probability of decode in threshold decode-and-forward multi-antenna relaying.

where $\lambda=g \sin ^{2}(\pi / M)$. For BPSK $M=2, h=1 / 2, g=1$, and equality is satisfied. For higher MPSK constellations $h=$ 1 and for other coherent binary transmission like orthogonal BFSK, $M=2, g=1 / 2, h=1 / 2$ [28]. In this paper, MPSK modulation schemes are used to illustrate the derivations, other coherent modulation schemes that have error rate defined by Q-function in AWGN can be accommodated as well.

Let us express the combined received SNR in this general form

$$
\gamma=\sum_{i=1}^{L_{c}} \gamma_{i},
$$

where $L_{c}=L=1$ implies no diversity,

$L_{c}=1, L>1$ implies SC diversity,

$L_{c}=L, L>1$ implies MRC diversity, and finally,

$1<L_{c} \leq L, L>1$ represents the generalized selection combining (GSC) diversity.

Traditionally, BER is computed by averaging the conditional BER (i.e., (16)) over the underlying PDF. Following this trend, the error rate for a relay that blindly decodes and forwards (i.e., does not perform threshold detection) is

$$
P_{e}=\int_{0}^{\infty} P_{\operatorname{mpsk}}(e \mid \gamma) p_{\gamma}(\gamma) d \gamma
$$

In the threshold decoding the receiver refrains from detection if the received SNR falls below the threshold $\gamma_{t h}$. The probability of error at the relay is thus the probability of error in the $\gamma \geq \gamma_{t h}$ regimes. Let us call this error performance $P_{e, r}\left(\bar{\gamma}, \gamma_{t h}\right)$. Proceeding with the integration in (18) we have

$$
\begin{aligned}
\int_{0}^{\infty} & P_{\mathrm{mpsk}}(e \mid \gamma) p_{\gamma}(\gamma) d \gamma \\
& =\int_{\gamma=0}^{\gamma_{t h}} P_{\mathrm{mpsk}}(e \mid \gamma) p_{\gamma}(\gamma) d \gamma \\
& +\int_{\gamma_{t h}}^{\infty} P_{\mathrm{mpsk}}(e \mid \gamma) p_{\gamma}(\gamma) d \gamma \\
& =\int_{\gamma=0}^{\gamma_{t h}} P_{\mathrm{mpsk}}(e \mid \gamma) p_{\gamma}(\gamma) d \gamma+v P_{e, r}\left(\bar{\gamma}, \gamma_{t h}\right),
\end{aligned}
$$

upon which $P_{e, r}\left(\bar{\gamma}, \gamma_{t h}\right)$ is expressed as

$$
\begin{aligned}
P_{e, r}\left(\bar{\gamma}, \gamma_{t h}\right) & =\frac{1}{v}(\underbrace{\int_{0}^{\infty} P_{\mathrm{mpsk}}(e \mid \gamma) p_{\gamma}(\gamma) d \gamma}_{I_{1}} \\
& -\underbrace{\int_{\gamma=0}^{\gamma_{t}} P_{\mathrm{mpsk}}(e \mid \gamma) p_{\gamma}(\gamma) d \gamma}_{I_{2}}) .
\end{aligned}
$$

Next, we evaluate $I_{1}$ and $I_{2}$ for the MRC-based detection at the multi-antenna relay. The factor $v$ has been evaluated in Appendix I and the derivation of $I_{1}$ and $I_{2}$ for SC-based detection is contained in Appendix II.

\section{A. MRC-Based Multi-Antenna Relay and Threshold Decode- and-Forward Strategy}

First, we provide the performance expressions for the scenario where MRC-based TDF relaying technique. In this case, using the PDF in (14), $I_{1}$ in (20) is obtained thus,

$$
\begin{aligned}
I_{1}^{(M R C)} & =h \int_{0}^{\infty}\left(\frac{m}{\bar{\gamma}}\right)^{L m} \frac{\gamma^{L m-1}}{\Gamma(L m)} \\
& \times \exp \left(-\frac{m \gamma}{\bar{\gamma}}\right) \operatorname{erfc}(\sqrt{\gamma \lambda}) d \gamma,
\end{aligned}
$$

where this integral can be expressed in terms of generalized hypergeometric function as

$$
\begin{aligned}
I_{1}^{(M R C)} & =\frac{h \Gamma[L m+1 / 2]}{\sqrt{\pi} \Gamma[L m+1]}\left(\frac{m}{\bar{\gamma} \lambda}\right)^{L m} \\
& \times{ }_{2} F_{1}\left[L m, L m+1 / 2 ; L m+1 ;-\frac{m}{\lambda \bar{\gamma}}\right] .
\end{aligned}
$$

Further simplification can be performed by using [29, pp. $1069(9.131 .1)]$ to express ${ }_{2} F_{1}\left[L m, L m+1 / 2 ; L m+1 ; \frac{-m}{\lambda \bar{\gamma}}\right]$ as $\left(\frac{\lambda \bar{\gamma}}{\lambda \bar{\gamma}+m}\right)^{L m}{ }_{2} F_{1}\left[L m, 1 / 2, L m+1, \frac{m}{\lambda \bar{\gamma}+m}\right]$. Finally, using [29, pp. 960 (8.391)] (22) can be simplified to

$$
I_{1}^{(M R C)}=\frac{h \Gamma[L m+1 / 2]}{\sqrt{\pi} \Gamma[L m]} B_{\mu}[L m, 1 / 2] .
$$

This expression is more compact than the one shown in [31].

To evaluate $I_{2}$ we follow the same steps as performed above

$$
\begin{aligned}
I_{2}^{(M R C)} & =h \int_{0}^{\gamma_{t h}}\left(\frac{m}{\bar{\gamma}}\right)^{L m} \frac{\gamma^{L m-1}}{\Gamma[L m]} \\
& \times \exp \left(-\frac{m \gamma}{\bar{\gamma}}\right) \operatorname{erfc}(\sqrt{\gamma \lambda}) d \gamma .
\end{aligned}
$$

It is difficult to obtain a close form expression for the exact integral of (24). Therefore, two things can be done. The first is to employ numerical integration techniques to evaluate it. The second option is to invoke some approximations that are known to be tight. For example $1 / 2 \operatorname{erfc}(x / \sqrt{2})$ has been shown to be well approximated by $\exp \left(-x^{2} / 2\right) /(\sqrt{2 \pi} x), x>$ 0 [30]. Therefore, (24) can be expressed as

$$
\begin{aligned}
I_{2}^{(M R C)} & \approx \frac{h}{\sqrt{\pi \lambda \gamma}} \int_{0}^{\gamma_{t h}}\left(\frac{m}{\bar{\gamma}}\right)^{L m} \frac{\gamma^{L m-1}}{\Gamma[L m]} \\
& \times \exp \left(-\left(\frac{m \gamma}{\bar{\gamma}}+\lambda \gamma\right)\right) d \gamma,
\end{aligned}
$$


where a convenient expression can then be obtained as

$$
\begin{aligned}
I_{2}^{(M R C)} & \approx \frac{h\left(\frac{m}{\bar{\gamma}}\right)^{L m}}{\sqrt{\pi \lambda} \Gamma[L m]\left(\lambda+\frac{m}{\bar{\gamma}}\right)^{L m-1 / 2}}(\Gamma[L m-1 / 2] \\
& \left.-\Gamma\left[L m-1 / 2, \lambda \gamma_{t h}+\frac{m \gamma_{t h}}{\bar{\gamma}}\right]\right)
\end{aligned}
$$

where $\Gamma[a, z]=\int_{z}^{\infty} t^{a-1} e^{-t} d t$ represents the upper incomplete gamma function. Though, this approximation is tight at high SNR, it is loose at low SNR. In certain scenarios, the low SNR regime may be of interest. Therefore, the following exact expression for low SNR regime is pursued which can be combined with the high SNR regime analysis above for evaluating the system performance. It is noted that $\operatorname{erfc}(x)=$ $\Gamma\left[1 / 2, x^{2}\right] / \sqrt{\pi}$. Therefore, $\operatorname{erfc}(\sqrt{\gamma \lambda})=\Gamma[1 / 2, \gamma \lambda] / \sqrt{\pi}$ and by using [29] $I_{2}^{(M R C)}$ can be written as

$$
\begin{aligned}
I_{2}^{(M R C)} & =h \int_{0}^{\gamma_{t h}}\left(\frac{m}{\bar{\gamma}}\right)^{L m} \frac{\gamma^{L m-1}}{\Gamma(L m)} \exp \left(-\frac{m \gamma}{\bar{\gamma}}\right) \\
& \times\left(1-\frac{2}{\sqrt{\pi}} \sum_{n=0}^{\infty} \frac{(-1)^{n}}{n !(2 n+1)}(\lambda \gamma)^{n+1 / 2}\right) d \gamma
\end{aligned}
$$

with further manipulations, it can be shown that

$$
\begin{aligned}
I_{2}^{(M R C)} & =\frac{h \Gamma[L m]-\Gamma\left[L m, \frac{m \gamma_{t h}}{\bar{\gamma}}\right]}{\Gamma[L m]} \\
& -\frac{4 h}{\sqrt{\pi}} \sum_{p=0}^{\infty} \frac{(-1)^{p}}{(p !(2 p+1))} \frac{\lambda^{p+1 / 2}}{\Gamma[L m]\left(\frac{m}{\bar{\gamma}}\right)^{p+1 / 2}} \\
& \times(\Gamma[L m+p+1 / 2] \\
& \left.-\Gamma\left[L m+p+1 / 2, m \gamma_{t h} / \bar{\gamma}\right]\right) .
\end{aligned}
$$

Similarly, error performance expressions for the SC-based relaying can be derived. The steps are given in Appendix II.

\section{B. On the Selection of Threshold Value}

Fig. 3 shows the variation of the BER with the threshold for different network configurations and various values of average SNR (ASNR). The figure demonstrates that multiple antennas at relays can relieve the cooperation's dependence on the threshold decoding protocol, especially when there are few relays and many antennas at these relays. It is found that for large $L$ and small $N_{R}$, E2E performance is insensitive to the choice of threshold over a wide range of threshold values (Fig. 3). In this scenario, it is preferred that a threshold is not applied at all. For small $L$ and large $N_{R}$ the choice of threshold is critical. The results also indicate that a good choice for the threshold is $\gamma_{t h} \approx \sqrt{2 \bar{\gamma}[\mathrm{dB}]}$ which is used in [24] for a simple single antenna relay network. This setting does not generalize for the multi-antenna multi-relay. However, any reasonable threshold setting can be used to compare the system architectures as it provides some indications of their relative performance. Although, such a setting may not produce the best possible E2E performance.

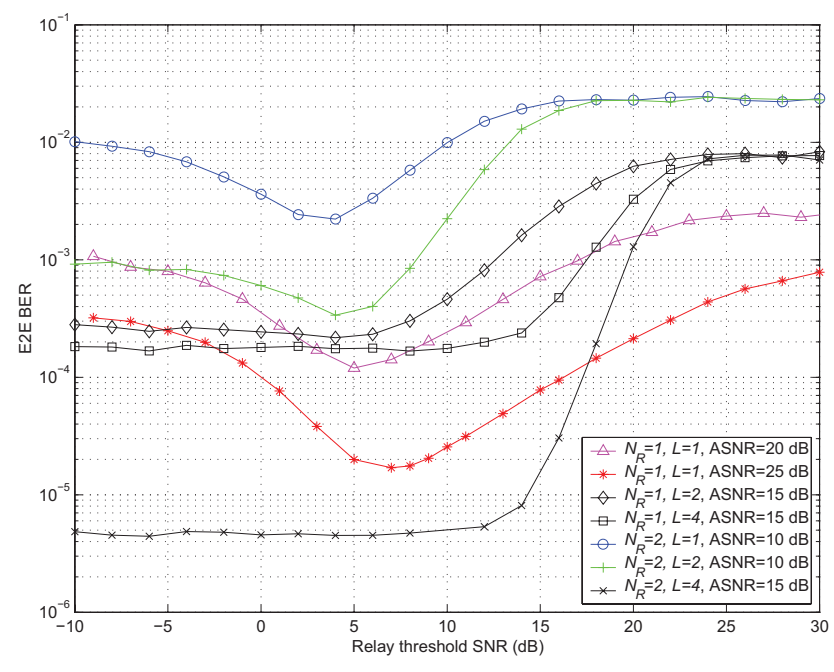

Fig. 3. E2E BER vs. relay decoding threshold for different network configurations and average SNR. $\left(N_{R}=1,2\right.$, and $\left.L=2,4\right)$.

The issue of optimum threshold remains an open problem as it has not received sufficient attention. For instance, recently, it has been shown that in asymmetric relay networks the choice of threshold depends on S-D and R-D channels [32] in contrast to the S-R channels usually used in symmetric relay networks. Therefore, more investigations of this issue is required for any comprehensive conclusion.

\section{NUMERICAL ILLUSTRATIONS}

Fig. 4 shows the performance of the multi-antenna relay in symmetrical networks for different number of antennas $(L)$ at the relay. The relay utilizes MRC and BPSK modulation is used in all the links. Results are shown for $m=1,2,6$ and $L$ $=1,2,4$. It is assumed that the fading distributions between the tripartite (source to relay, source to destination and relay to source) are identical. The performance of conventional relaying (see network relay deployment, Fig. 1 (a)) is shown as well. The evaluation of system performance for non-identical fading conditions is straightforward. For instance, suppose a fixed relay is positioned in such a way that it sees NLOS to the source and LOS to the destination. Assuming that the source have NLOS to destination, the relay-destination channel can be modeled with a suitable $m$ using the Nakagami- $m$ to Ricean $K$-factor transformation and the source-relay and sourcedestination channels modeled using the Rayleigh distribution $(m=1)$.

From Fig. 4, it is observed that the threshold-based multiantenna relay systems yield significant gains over the referenced single antenna fixed protocol relaying. Let us compare the SNR requirements at an error rate of $10^{-2}$. The following approximate gains are obtained over the conventional relaying, $10.5 \mathrm{~dB}(L=1), 13 \mathrm{~dB}(L=2)$, and $14.5 \mathrm{~dB}(L=4)$ for the Rayleigh channels $(m=1)$. These gains represent a significant improvement. Furthermore, at BER $=10^{-3}$, the TDF protocol with dual antenna exhibits about $4 \mathrm{~dB}$ superiority over that using a single antenna. When four antennas are deployed, this gain is about $5 \mathrm{~dB}$. For $m=2$, however, the gain of dual antennas over the single antenna is about $1.5 \mathrm{~dB}$ while that of four antennas is $2.5 \mathrm{~dB}$. From these cases, it can be 


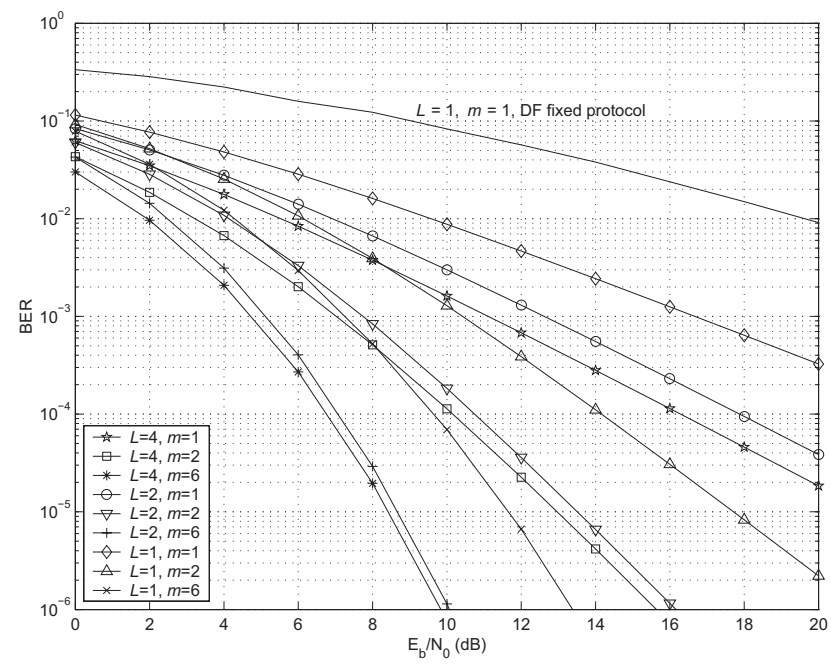

Fig. 4. BER performance of MRC-based multi-antenna TDF relay in Nakagami fading, $N_{R}=1$.

TABLE I

MRC-BASED AND SC-BASED RELAY DETECTION: REQUIRED SNR FOR A BER $=10^{-4}$ FOR DIFFERENT NETWORK SCENARIOS

\begin{tabular}{||c|c|c|c|c||}
\hline \hline MRC-based & \multicolumn{2}{|c|}{$N_{R}=2$} & \multicolumn{2}{c|}{$N_{R}=4$} \\
\hline \# of antennas $L$ & $m=1$ & $m=2$ & $m=1$ & $m=2$ \\
\hline 1 & $18.0 \mathrm{~dB}$ & $11.5 \mathrm{~dB}$ & $12.8 \mathrm{~dB}$ & $9.2 \mathrm{~dB}$ \\
\hline 2 & $12.2 \mathrm{~dB}$ & $8.0 \mathrm{~dB}$ & $8.2 \mathrm{~dB}$ & $6.4 \mathrm{~dB}$ \\
\hline 4 & $10.4 \mathrm{~dB}$ & $6.8 \mathrm{~dB}$ & $5.8 \mathrm{~dB}$ & $3.8 \mathrm{~dB}$ \\
\hline SC-based & \multicolumn{2}{|c|}{$N_{R}=2$} & \multicolumn{2}{|c||}{$N_{R}=4$} \\
\hline \# of antennas $L$ & $m=1$ & $m=2$ & $m=1$ & $m=2$ \\
\hline 1 & $18.0 \mathrm{~dB}$ & $11.5 \mathrm{~dB}$ & $13.0 \mathrm{~dB}$ & $9.2 \mathrm{~dB}$ \\
\hline 2 & $13.2 \mathrm{~dB}$ & $9.2 \mathrm{~dB}$ & $9.2 \mathrm{~dB}$ & $7.6 \mathrm{~dB}$ \\
\hline 4 & $10.8 \mathrm{~dB}$ & $7.8 \mathrm{~dB}$ & $7.6 \mathrm{~dB}$ & $6.1 \mathrm{~dB}$ \\
\hline \hline
\end{tabular}

deduced that the diversity gain is better for the multi-antenna system in Rayleigh $(m=1)$ than in less scattering channels $(m>1)$ which again confirms the notion of the benefit of the rich scattering environment. Furthermore, with dual antennas, the necessary diversity is already acquired, and only marginal gains are observed for an increasing number of antennas.

Fig. 5 shows the performance of the network with $N_{R}=$ 2. We compare this relay network for different number of antennas at a BER $=10^{-4}$. In Rayleigh fading, it is observed that a gain of $6 \mathrm{~dB}$ is achieved for the network $\left[N_{R}=2, L=\right.$ $2]$ over that of $\left[N_{R}=2, L=1\right]$. The gain of the network $\left[N_{R}=2, L=4\right]$ over that of $\left[N_{R}=2, L=1\right]$ increases only marginally to $7.5 \mathrm{~dB}$ which buttresses the point that with dual antenna, the necessary gain is almost derived. For the less severe fading cases $(m>1)$, we observe that the gains are reduced. For example, for $m=2$, a gain of $3.5 \mathrm{~dB}$ is obtained for configuration $\left[N_{R}=2, L=2\right]$ and $4.7 \mathrm{~dB}$ for [ $\left.N_{R}=2, L=4\right]$ over that of $\left[N_{R}=2, L=1\right]$. The trends observed in Figs. 4 and 5 are generally seen for the multiantenna 4-relay network. The performance of this network is shown in Fig. 6. Let us now compare some of the system architectures (Table I and Fig. 7). In the table the SNR required for an error rate $10^{-4}$ for different $L$ and $m$ is considered. It is observed that $\left[N_{R}=2, L=2\right]$ network performs comparably to that $\left[N_{R}=4, L=1\right]$, although, the number of detection chains required in both configurations is the same. However,

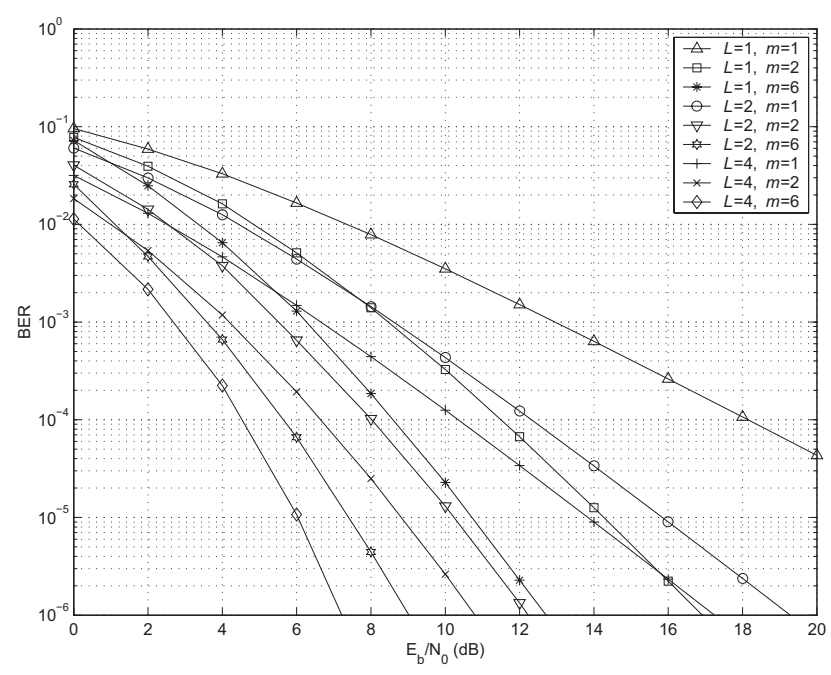

Fig. 5. BER performance of MRC-based multi-antenna TDF relay in Nakagami fading, $N_{R}=2$.

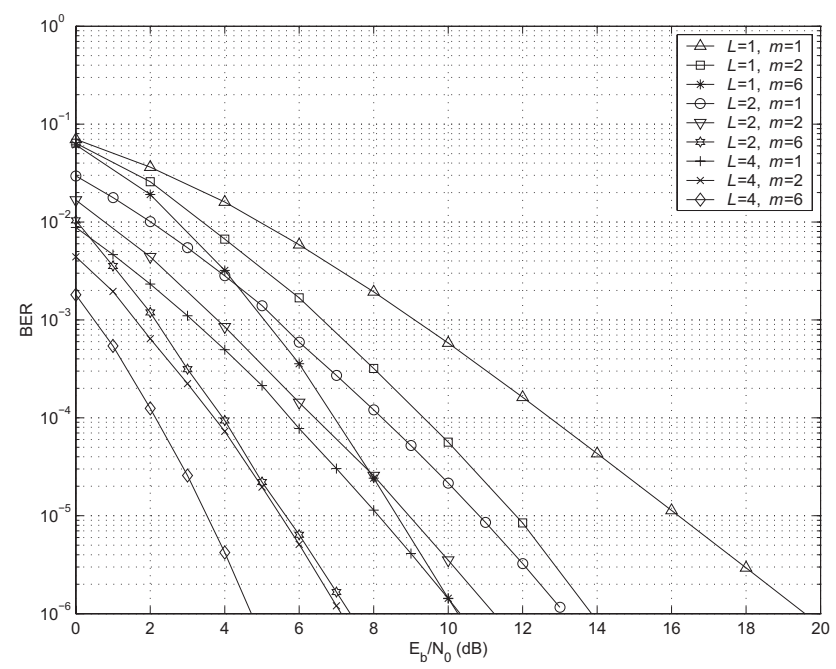

Fig. 6. BER performance of MRC-based multi-antenna TDF relay in Nakagami fading, $N_{R}=4$.

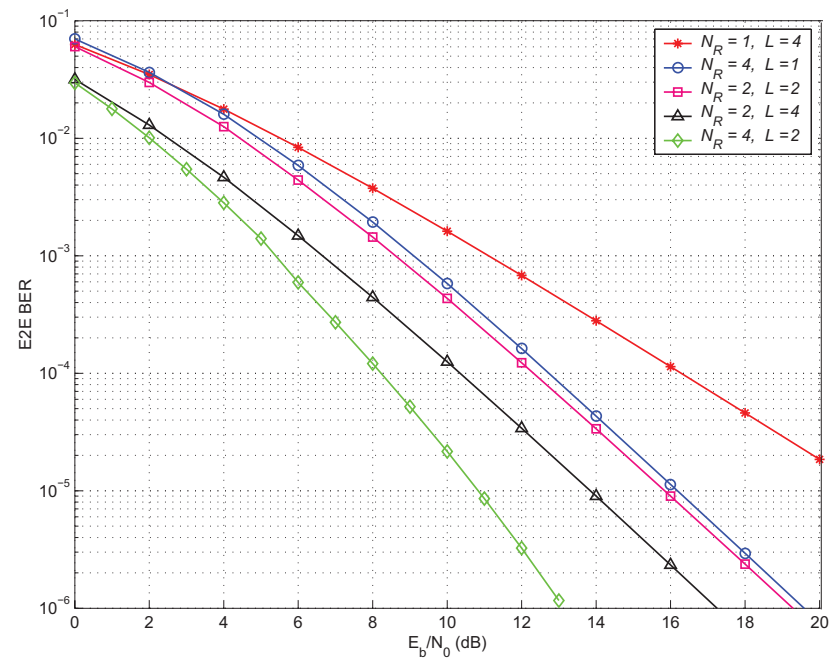

Fig. 7. Comparison of network architectures demonstrating the impact of multiple antennas on relays in Rayleigh fading. 
the network $\left[N_{R}=2, L=2\right]$ has an additional advantage over that of $\left[N_{R}=4, L=1\right]$; it is considerably cheaper to install additional antennas at relays than deploying more relays. When two antennas are deployed with $N_{R}=4$, it is observed that $\left[N_{R}=4, L=2\right]$ provides a SNR gain over [ $\left.N_{R}=2, L=4\right]$ of about $2.2 \mathrm{~dB}$ for $m=1$ and about 0.4 $\mathrm{dB}$ for $(m=2)$. The gain of the network $\left[N_{R}=4, L=2\right]$ over that of $\left[N_{R}=2, L=4\right]$ has to be carefully viewed. If the cost of these two systems is considered (deploying two relay stations as opposed to installing two antennas on relay stations) this gain as inconsequential can be quickly dismissed, i.e., it is too small in comparison to the concomitant cost and complexity over the network $\left[N_{R}=2, L=4\right]$. In fact, as observed, this gain disappears at a less severe fading. In conclusion, the network $\left[N_{R}=2, L=4\right]$ maybe preferred to that of [ $\left.N_{R}=4, L=2\right]$ for deployment purposes considering the capital involvement on land acquisition, labor, maintenance, signalling overhead, radio resources, etc. This conclusion is based on the fact that cooperative diversity is the main interest. In addition, the impact of deploying four antennas on a single relay node as opposed to four relays nodes each with single antenna should be noted (Fig. 7). Note also that without these results, intuition would have led to the belief that the diversity order for the $N_{R}=4$ network is five $\left(N_{R}=4+1\right)$. The fact is that it only has the potential of providing this much diversity order. Clearly, Fig. 7 shows that this is not the case. The configuration $\left[N_{R}=2, L=4\right]$ indicates that adding more antennas (from $L=2$ to $L=4$ ) does not provide more diversity order; however, it will only increase antenna gains. Hence, an important conclusion that can be derived is that two antennas are enough to provide the maximum diversity order, but any additional antenna provides only antenna gains. This also helps explain why [ $\left.N_{R}=2, L=2\right]$ shows performance advantage over the setup $\left[N_{R}=4, L=1\right]$. Moreover, increasing the number of antennas, the $N_{R}=2$ network can significantly outperform $\left[N_{R}=4, L=1\right]$. At BER $=10^{-5},\left[N_{R}=2, L=4\right]$ provides more that $2 \mathrm{~dB}$ SNR gain over $\left[N_{R}=4, L=1\right]$.

Fig. 8 serves to compare the threshold-based MRC and $\mathrm{SC}$ relaying. First, let us compare these techniques for the Rayleigh fading. Fig.s 8 (a), (b), (c), and (d) show that MRCbased relay detection could yield no more than $2 \mathrm{~dB}$ gain over the SC-based. The maximum gain is recorded for a large system configuration, specifically with $\left[N_{R}=4, L=4\right]$. For a smaller number of relays (say $N_{R}=2$ ), the gain considerably shrinks. Furthermore, it is observed that though MRC-based relaying is about $2 \mathrm{~dB}$ superior to the SC-based counterpart the former is at expense of a huge system complexity and cost in the network $\left[N_{R}=4, L=4\right]$. For instance, the MRC requires sixteen separate receiver chains (four at each relay station) while the SC-based requires only four. The low SNR gain cannot offset the cost disadvantage incurred by using MRC. Therefore, SC-based relaying offers an excellent tradeoff between cost and performance. Finally, for higher values of Nakagami parameter $(m>1)$, MRC still records gains over the SC-based detection but these gains are too small to justify for the cost involved. This, further, tilts the balance in favor of SC-based detection at relays.

We will now compare the SC-based detection for different system configurations (Table I). We consider the SNR required for an error rate of $10^{-4}$ for different $L$ and $m$. We compare network configurations [ $\left.N_{R}=4, L=1\right],\left[N_{R}=4, L=2\right]$, and $\left[N_{R}=2, L=4\right]$. In the case of the networks $\left[N_{R}=\right.$ $4, L=1]$ and $\left[N_{R}=4, L=2\right]$, four detection chains are required but the network $\left[N_{R}=4, L=2\right]$ requires extra four inexpensive antennas and switching mechanisms. It is observed that in Rayleigh fading environments, a gain as high as $3.8 \mathrm{~dB}$ is obtained with the network $\left[N_{R}=4, L=2\right]$ over that of $\left[N_{R}=4, L=1\right]$ and this gain drops to $1.6 \mathrm{~dB}$ for $m=2$. When $\left[N_{R}=2, L=4\right]$ network configuration is employed in place of $\left[N_{R}=4, L=2\right]$, a degradation of 1.6 and $0.2 \mathrm{~dB}$, for $m=1$ and $m=2$, respectively, is observed. However, two detection chains are used in $\left[N_{R}=2, L=4\right]$ network as compared to four in $\left[N_{R}=4, L=2\right]$ network. Furthermore, as Fig. 8 and Table I indicate, deploying two relays each with two antennas $\left[N_{R}=2, L=2\right]$ with $\mathrm{SC}$ at the relay yields almost the same performance as deploying four relays each with one antenna $\left[N_{R}=4, L=1\right]$ (Fig. 6). It is worth mentioning that two detection resources are required in the $\left[N_{R}=2, L=2\right]$ case whereas the $\left[N_{R}=4, L=1\right]$ case necessitates the use of four detection resources. Besides, the resource required for deploying extra relays is not comparable to microdiversity antenna elements. Therefore, deploying microdiversity at relays may result in considerable savings in the number of relays to be deployed in a given area.

\section{CONCLUSION}

This paper investigates the cooperative diversity achieved when multiple relays and source are engaged in a cooperation in two-hop wireless networks. Since space, cost, and signal processing constraints prohibit the use of a large number of antennas at wireless terminals, the promises associated with multi-antenna techniques can be exploited through such a cooperation by mimicking the performance of a large array of antennas.

Infrastructure-based fixed relays may have the capability to carry multiple antennas in contrast to terminals. Therefore, multi-antenna relay networks are examined. Threshold-based maximal ratio combining and threshold-based selection combining techniques to diversity process the relay signals are analyzed. From the perspective of end-to-end error performance, it is derived that for a given performance requirement, the multiple antennas at relays can significantly reduce the number of relays required in a network area, with a considerable impact on the system deployment investment. The threshold selection combining relaying emerges as an excellent candidate in terms of performance-complexity tradeoff. Furthermore, it is observed that two antennas at the relays are enough to provide the diversity order equal to the number of relays in the network plus one. It is also found that threshold decoding is not required when there are few relays and these relays have large number antennas.

\section{APPENDIX I}

\section{NORMALIZING FACTOR}

For MRC-based relaying, the normalizing factor is

$$
v_{M R C}=\int_{\gamma_{t h}}^{\infty}\left(\frac{m}{\bar{\gamma}}\right)^{L m} \frac{\gamma^{L m-1}}{\Gamma[L m]} \exp \left(-\frac{m \gamma}{\bar{\gamma}}\right) d \gamma .
$$




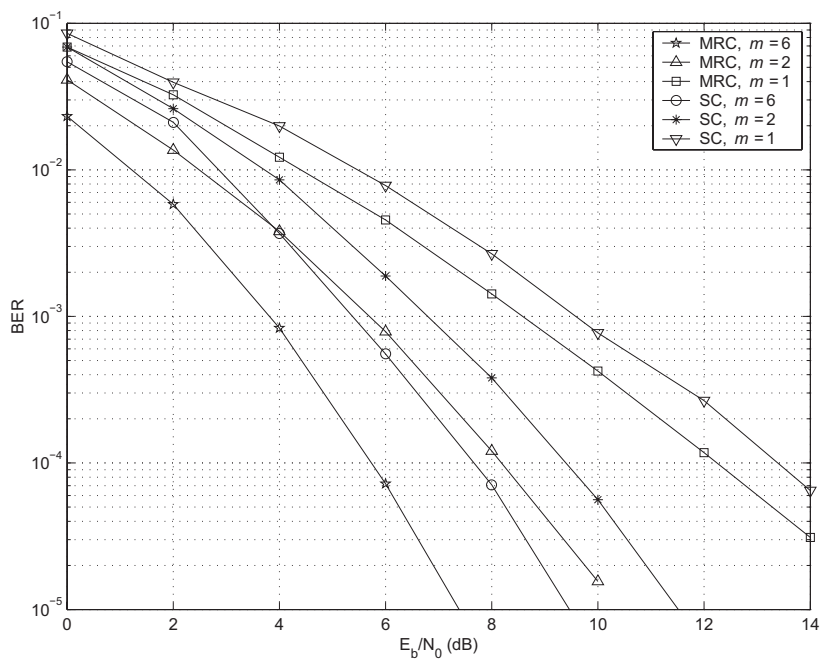

(a) $N_{R}=2, L=2$

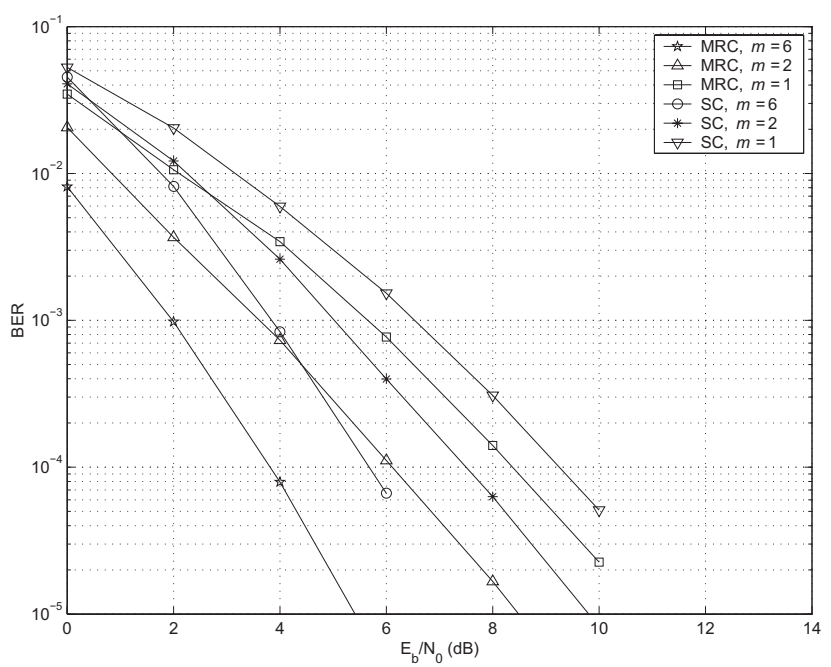

(c) $N_{R}=4, L=2$

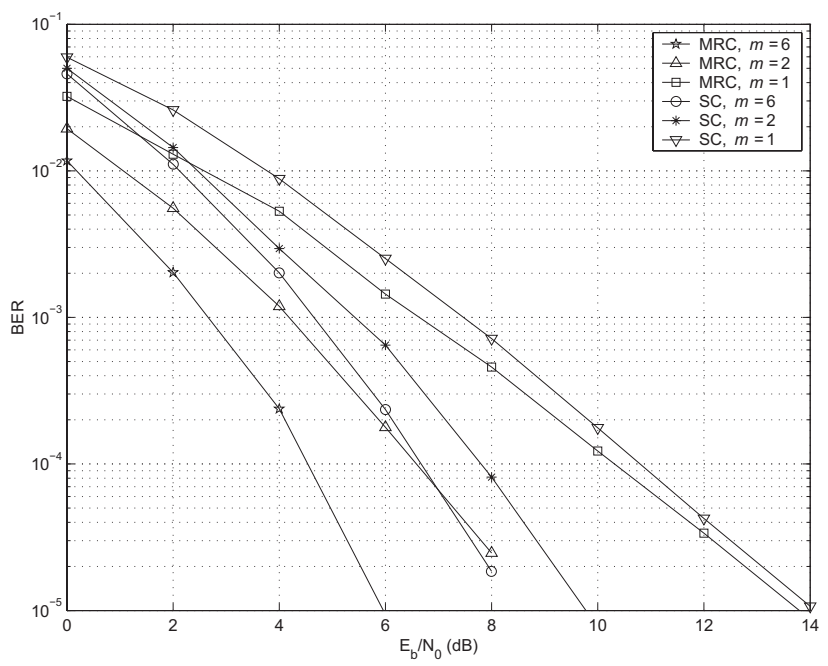

(b) $N_{R}=2, L=4$

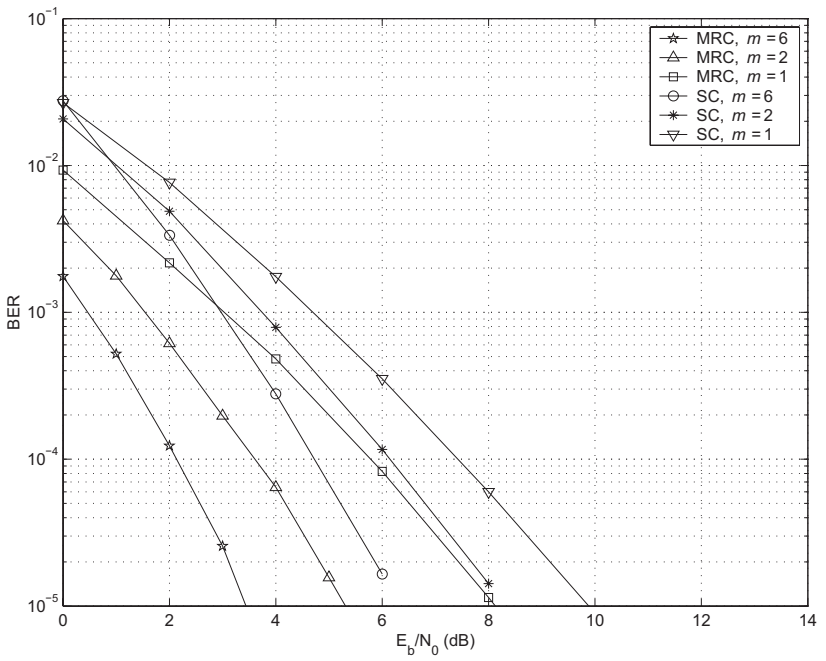

(d) $N_{R}=4, L=4$

Fig. 8. Performance comparison of MRC-based vs. SC-based multi-antenna TDF relay in Nakagami fading.

With the definition of the incomplete gamma function the expression in (29) can be expressed as

$$
v_{M R C}=\frac{\Gamma\left[L m, \frac{m \gamma_{t h}}{\bar{\gamma}}\right]}{\Gamma[L m]} .
$$

For Rayleigh fading $(m=1)$ and no diversity antennas at relay (i.e., $L=1$ ),

$$
v=\Gamma\left[L m, \frac{m \gamma_{t h}}{\bar{\gamma}}\right]=\Gamma\left[1, \gamma_{t h} / \bar{\gamma}\right]=\exp \left(-\gamma_{t h} / \bar{\gamma}\right)
$$

For Nakagami SC-based relaying, the normalizing factor is

$$
\begin{aligned}
v_{S C} & =\int_{\gamma_{t h}}^{\infty} \frac{L}{(m-1) !} \sum_{l=0}^{L-1}(-1)^{l}\left(\begin{array}{c}
L-1 \\
l
\end{array}\right) \sum_{k=0}^{l(m-1)} b_{k}^{l} \\
& \times\left(\frac{m}{\bar{\gamma}}\right)^{m+k} \gamma^{m+k-1} \exp \left(-(l+1) \frac{m \gamma}{\bar{\gamma}}\right) d \gamma, \\
& =\frac{L}{(m-1) !} \sum_{l=0}^{L-1}(-1)^{l}\left(\begin{array}{c}
L-1 \\
l
\end{array}\right) \sum_{k=0}^{l(m-1)} b_{k}^{l}
\end{aligned}
$$

$$
\times\left(\frac{1}{1+l}\right)^{k+m} \Gamma\left[k+m,(1+l) \frac{m \gamma_{t h}}{\bar{\gamma}}\right] .
$$

\section{APPENDIX II}

SC-BASED Multi-AnTENNA RELAY AND THRESHOLD DECODE-AND-FORWARD STRATEGY

The derivation of error expressions for SC-based relay is considered here. In this scenario, the PDF in (12) is employed in computing $I_{1}^{(S C)}$, thus

$$
\begin{aligned}
I_{1}^{(S C)} & =h \int_{0}^{\infty} \frac{L}{(m-1) !} \sum_{l=0}^{L-1}(-1)^{l}\left(\begin{array}{c}
L-1 \\
l
\end{array}\right) \\
& \times \sum_{k=0}^{l(m-1)} b_{k}^{l}\left(\frac{m}{\bar{\gamma}}\right)^{m+k} \gamma^{m+k-1} \\
& \times \exp \left(-(l+1) \frac{m \gamma}{\bar{\gamma}}\right) \operatorname{erfc}(\sqrt{\gamma \lambda}) d \gamma, \\
& =\frac{h L}{(m-1) !} \sum_{l=0}^{L-1}(-1)^{l}\left(\begin{array}{c}
L-1 \\
l
\end{array}\right) \sum_{k=0}^{l(m-1)} b_{k}^{l}
\end{aligned}
$$




$$
\begin{aligned}
& \times \quad\left(\frac{m}{\bar{\gamma}}\right)^{m+k} \int_{0}^{\infty} \gamma^{m+k-1} \exp \left(-(l+1) \frac{m \gamma}{\bar{\gamma}}\right) \\
& \times \quad \operatorname{erfc}(\sqrt{\gamma \lambda}) d \gamma
\end{aligned}
$$

Using the same approach as employed for MRC, $I_{1}^{(S C)}$ can be expressed as

$$
\begin{aligned}
I_{1}^{(S C)} & =\frac{h L}{(m-1) !} \sum_{l=0}^{L-1}(-1)^{l}\left(\begin{array}{c}
L-1 \\
l
\end{array}\right) \sum_{k=0}^{l(m-1)} b_{k}^{l} \\
& \times\left(\frac{m}{\bar{\gamma} \lambda}\right)^{m+k} \frac{\Gamma[1 / 2+k+m]}{\sqrt{\pi}(k+m)}{ }_{2} F_{1}[k+m, k+m \\
& \left.+1 / 2 ; k+m+1 ; \frac{-m(1+l)}{\lambda \bar{\gamma}}\right] .
\end{aligned}
$$

With the help of [29], the hypergeometric function can be expressed as ${ }_{2} F_{1}\left[k+m, k+m+1 / 2 ; k+m+1 ; \frac{-m(1+l)}{\lambda \bar{\gamma}}\right]=$ $\left(\frac{\lambda \bar{\gamma}+m(1+l)}{\lambda \bar{\gamma}}\right)^{-(k+m)}{ }_{2} F_{1}\left[k+m, 1 / 2, k+m+1, \frac{m(1+l)}{m(1+l)+\lambda \bar{\gamma}}\right]$, thus, employing [29, pp. 960, (8.391)] a simplified expression is obtained

$$
\begin{aligned}
I_{1}^{(S C)} & =\frac{h L}{\sqrt{\pi}(m-1) !} \sum_{l=0}^{L-1}(-1)^{l}\left(\begin{array}{c}
L-1 \\
l
\end{array}\right) \sum_{k=0}^{l(m-1)} b_{k}^{l} \\
& \times \frac{\Gamma[k+m+1 / 2]}{(1+l)^{k+m}} B_{y}[k+m, 1 / 2],
\end{aligned}
$$

where $y=\frac{m(1+l)}{\lambda \bar{\gamma}+m(1+l)}$.

Finally, the derivation of $I_{2}^{(S C)}$ is performed as

$$
\begin{aligned}
I_{2}^{(S C)} & =h \int_{0}^{\gamma_{t h}} \frac{L}{(m-1) !} \sum_{l=0}^{L-1}(-1)^{l}\left(\begin{array}{c}
L-1 \\
l
\end{array}\right) \\
& \times \sum_{k=0}^{l(m-1)} b_{k}^{l}\left(\frac{m}{\bar{\gamma}}\right)^{m+k} \gamma^{m+k-1} \\
& \times \exp \left(-(l+1) \frac{m \gamma}{\bar{\gamma}}\right) \operatorname{erfc}(\sqrt{\gamma \lambda}) d \gamma \\
& \approx \frac{h L}{(m-1) !} \sum_{l=0}^{L-1}(-1)^{l}\left(\begin{array}{c}
L-1 \\
l
\end{array} \sum_{k=0}^{l(m-1)} b_{k}^{l}\right. \\
& \times\left(\frac{m}{\bar{\gamma}}\right)^{m+k} \frac{1}{\sqrt{\pi \lambda}\left(\lambda+\frac{m(l+1)}{\bar{\gamma}}\right)^{m+k-1 / 2}} \\
& \times(\Gamma[m+k-1 / 2] \\
& \left.-\Gamma\left[m+k-1 / 2, \lambda \gamma_{t h}+\frac{m(l+1) \gamma_{t h}}{\bar{\gamma}}\right]\right) .
\end{aligned}
$$

As with (26) for MRC, this approximation is only tight at high SNR. Therefore, it is necessary to find an expression for the low SNR regimes. The steps to the final solution are almost the same as in the MRC derivation. Without repeating those steps, the final expression is given as

$$
\begin{aligned}
I_{2}^{(S C)} & =h \int_{0}^{\gamma_{t h}} \frac{L}{(m-1) !} \sum_{l=0}^{L-1}(-1)^{l}\left(\begin{array}{c}
L-1 \\
l
\end{array}\right) \\
& \times \sum_{k=0}^{l(m-1)} b_{k}^{l}\left(\frac{m}{\bar{\gamma}}\right)^{m+k} \gamma^{m+k-1} \\
& \times \exp \left(-(l+1) \frac{m \gamma}{\bar{\gamma}}\right) \operatorname{erfc}(\sqrt{\gamma \lambda}) d \gamma \\
& =\frac{h L}{(m-1) !} \sum_{l=0}^{L-1}(-1)^{l}\left(\begin{array}{c}
L-1 \\
l
\end{array} \sum_{k=0}^{l(m-1)} b_{k}^{l}\right. \\
& \times\left(\frac{1}{1+l}\right){ }^{(k+m)}(\Gamma[k+m] \\
& -\Gamma\left[k+m, \frac{m(1+l) \gamma_{t h}}{\bar{\gamma}}\right] \\
& -\frac{4 h}{\sqrt{\pi} \sum_{p=0}^{\infty} \frac{(-1)^{p}}{p !(2 p+1)}\left(\frac{m}{\lambda \bar{\gamma}}\right)^{-(p+1 / 2)}} \\
& \times \frac{1}{(1+l)^{p+1 / 2}(\Gamma[k+m+p+1 / 2]} \\
& \left.\left.\Gamma\left[k+m+p+1 / 2, m(1+l) \gamma_{t h} / \bar{\gamma}\right]\right)\right) .
\end{aligned}
$$

\section{REFERENCES}

[1] J. Laneman, D. Tse, and G. Wornell, "Cooperative diversity in wireless networks: Efficient protocols and outage behavior," IEEE Trans. Inform. Theory, vol. 50, no. 11, pp. 3062-3080, Dec. 2004.

[2] A. Sendonaris, E. Erkip, and B. Aazhang, "User cooperation diversity - Part I: System description,” IEEE Trans. Commun., vol. 51, no. 11, pp. 1927-1938, Nov. 2003.

[3] G. J. Foschini and M. J. Gans, "On limits of wireless communications in fading environment when using multiple antennas," IEEE Wireless Pers. Commun., vol. 6, pp. 311-335, Mar. 1998.

[4] L. Zheng and D. N. C. Tse, "Diversity and multiplexing: A fundamental tradeoff in multiple antenna channels," IEEE Trans. Inform. Theory, vol. 49, no. 5, pp. 1073-1096, May 2003.

[5] E. Telatar, "Capacity of multi-antenna Gaussian channels," European Trans. Telecommun., vol. 6, pp. 585-595, Nov. 1999.

[6] J. Laneman and G. Wornell, "Distributed space-time-coded protocols for exploiting cooperative diversity in wireless networks," IEEE Trans. Inform. Theory, vol. 49, no. 10, pp. 2415-2425, Oct. 2003.

[7] R. Pabst, B. Walke, D. Schultz, P. Herhold, H. Yanikomeroglu, S. Mukherjee, H. Viswanathan, M. Lott, W. Zirwas, M. Dohler, H. Aghvami, D. Falconer, and G. Fettweis, "Relay-based deployment concepts for wireless and mobile broadband radio," IEEE Commun. Mag., pp. 80-89, Sep. 2004.

[8] H. Hu, H. Yanikomeroglu, D. Falconer, and S. Periyalwar, "Range extension without capacity penalty in cellular networks with digital fixed relays," in Proc. IEEE Globecom, Nov. 2004, vol. 5, pp. 3053-3057.

[9] V. Morgenshtern and H. Bölcskei, "On the value of cooperation in interference relay networks," in CD Record Allerton Conference, Dec. 2005.

[10] J. Boyer, H. Yanikomeroglu, and D. Falconer, "Multihop diversity in wireless relaying channels," IEEE Trans. Commun., vol. 52, no. 10, pp. 1820-1830, Oct. 2004

[11] M. Hasna and M. Alouini, "End-to-end performance of transmission systems with relays over Rayleigh fading channels," IEEE Trans. Wireless Commun., vol. 2, no. 6, pp. 1126-1131, Nov. 2003.

[12] M. Yuksel and E. Erkip, "Diversity in relaying protocols with amplify and forward," in Proc. IEEE Globecom, Dec. 2003, vol. 4, pp. 20252029.

[13] A. Stefanov and E. Erkip, "Cooperative space-time coding for wireless networks," IEEE Inform. Theory Workshop, Apr. 2003, pp. 50-53.

[14] Y. Hua, Y. Mei, and Y. Chang, "Parallel wireless mobile relays with space-time modulations," in Proc. IEEE Workshop Statistical Signal Processing, Oct. 2003, pp. 375-378. 
[15] T. Hunter and A. Nosratinia, "Cooperation diversity through coding," in Proc. IEEE Int'l Symp. Inform. Theory, June 2002, p. 220.

[16] E. Meulen, "Three-terminal communication channels," Adv. Appl. Probability, vol. 3, no. 1, pp. 120-154, Spring 1971.

[17] T. Cover and A. El-Gamal, "Capacity theorems for the relay channel," IEEE Trans. Inform. Theory, vol. 25, no. 5, pp. 572-584, Sep. 1979.

[18] IST-2003-507581 WINNER, "D3.4 Definition and assessment of relay based cellular deployment concepts for future radio scenarios considering 1st protocol characteristics." [Online.] Available: www.istwinner.org/DeliverableDocuments/D3-4.pdf

[19] M. Simon and M. Alouini, Digital Communication over Fading Channels: A Unified Approach to Performance Analysis. New York: John Wiley \& Sons, 2000.

[20] R. Braun and U. Dersch, "A physical mobile radio channel model," IEEE Trans. Veh. Technol., vol. 40, pp. 472-482, May 1991.

[21] A. Wittneben, "Basestation modulation diversity for digital SIMULCAST," in Proc. IEEE Veh. Technol. Conf., May 1991, pp. 848-853.

[22] A. Wittneben, "A new bandwidth efficient transmit antenna modulation diversity scheme for linear digital modulation," in Proc. IEEE Int'l Conf. Commun., May 1993, vol. 3, pp. 1630-1634.

[23] Y. Tu and G. Pottie, "Coherent cooperative transmission from multiple adjacent antennas to a distant stationary antenna through AWGN channels," IEEE Veh. Technol. Conf., May 2002, vol. 1, pp. 130-134.

[24] P. Herhold, E. Zimmermann, and G. Fettweis, "A simple cooperation extension to wireless relaying," in Proc. International Zurich Seminar Commun., Feb. 2004, pp. 36-39.

[25] A. Papoulis, Probability, Random Variables, and Stochastic Processes. New York: McGraw-Hill, 1991.

[26] G. Fedele, "Error probability for diversity detection of binary signals over Nakagami fading channels," in Proc. IEEE Int'l Conf. Pers. Indoor Mobile Radio Commun., Sep. 1994, vol. 2, pp. 609-611.

[27] E. Al-Hussaini and A. Al-Bassiouni, "Performance of MRC diversity systems for the detection of signals in Nakagami fading," IEEE Trans. Commun., vol. 33, pp. 1315-1319, Feb. 1985.

[28] J. Proakis, Digital Communications, New York: McGraw Hill, 1995.

[29] I. Gradshteyn and I. Ryzhik, Table of Integrals, Series, and Products. San Diego: Academic Press, 1994.

[30] S. Verdu, Multiuser Detection. Cambridge, UK: Cambridge University Press, 1998.

[31] M. Alouini and M. Simon, "Performance of coherent recievers with hybrid SC/MRC over Nakagami-m fading channels," IEEE Trans. Veh. Technol., vol. 48, no. 4, pp. 1155-1164, July 1999.

[32] F. Atay, A. Adinoyi, Fan Yijia, H. Yanikomeroglu, and J. Thompson, "On the optimum threshold of digital cooperative relaying schemes," submitted to IEEE Wireless Commun. and Networking Conf., Mar. 2007.

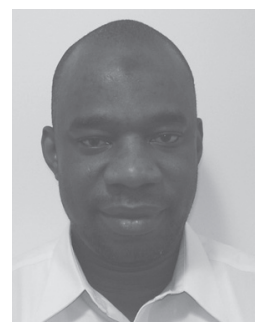

Abdulkareem Adinoyi received a B.Eng degree from the University of Ilorin, Nigeria, in 1992, M.S degree from the King Fahd University of Petroleum and Minerals (KFUPM), Dhahran, Saudi Arabia, in 1998 and Ph.D degree from Carleton University, Ottawa, Canada, in 2006, all in electrical engineering. He was with Dubi Oil Limited, Port Harcourt, Nigeria as an Instrument/Electrical Engineer from April 1993 to August 1995. He was with KFUPM between September 1995 and October 1998 as a Research Assistant. Between January 1999 and August 2002 he held the position of a lecturer at the Department of Electrical Engineering, KFUPM. He is currently a senior research associate at the Department of Systems and Computer Engineering at Carleton University where he participates in the European Union 6th Framework integrated project - the WINNER, dedicated to researching, developing and demonstrating a seamless multi-scenario next generation wireless air interface. His research interest is in wireless communication networks with a special emphasis on infrastructure-based multihop and relay networks, cooperative diversity schemes and protocols.

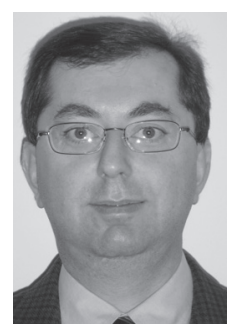

Halim Yanikomeroglu received a B.S. degree in electrical and electronics engineering from the Middle East Technical University, Ankara, Turkey, in 1990, and an M.A.S. degree in electrical engineering (now ECE), and a Ph.D. degree in electrical and computer engineering from the University of Toronto, Canada, in 1992 and 1998, respectively. He was with the Research and Development Group of Marconi Kominikasyon A.S., Ankara, Turkey, from January 1993 to July 1994 . Since 1998, he has been with the Department of Systems and Computer Engineering at Carleton University, Ottawa, where he is now an Associate Professor and Associate Chair for Graduate Studies. His research interests include almost all aspects of wireless communications with a special emphasis on infrastructure-based multihop/mesh/relay networks. He has been involved in the steering committees and technical program committees of numerous international conferences in communications; he has also given several tutorials in such conferences. He was the Technical Program Cochair of the IEEE Wireless Communications and Networking Conference 2004 (WCNC'04). He was an Editor for IEEE TRANSACTIONS ON WIRELESS COMMUNICATIONS during 2002 - 2005, and a Guest Editor for Wiley Journal on Wireless Communications \& Mobile Computing; he was an Editor for IEEE Communications SuRveys \& TUtorials for 2002 - 2003. Currently he is serving as the Chair of the IEEE Communications Society's Technical Committee on Personal Communications (TCPC), he is also a Member of IEEE ComSoc's Technical Activities Counsel (TAC). 